\title{
Adjoint Transient Sensitivity Analysis in Circuit Simulation
}

\author{
Z. Ilievski ${ }^{1}$, H. Xu ${ }^{1}$, A. Verhoeven ${ }^{1}$, E.J.W. ter Maten ${ }^{1,2}$, \\ W.H.A. Schilders ${ }^{1,2}$ and R.M.M. Mattheij ${ }^{1}$ \\ 1 Technische Universiteit Eindhoven; e-mail: Z.Ilievski@tue.nl \\ 2 NXP Semiconductors Eindhoven
}

Summary. Sensitivity analysis is an important tool that can be used to assess and improve the design and accuracy of a model describing an electronic circuit. Given a model description in the form of a set of differential-algebraic equations it is possible to observe how a circuit's output reacts to varying input parameters, which are introduced at the requirements stage of design. In this paper we consider the adjoint method more closely. This method is efficient when the number of parameters is large. We extend the transient sensitivity work of Petzold et al., in particular we take into account the parameter dependency of the dynamic term. We also compare the complexity of the direct and adjoint sensitivity and derive some error estimates. Finally we sketch out how Model Order Reduction techniques could be used to improve the efficiency of adjoint sensitivity analysis.

Keywords - Sensitivity Analysis; Transient Analysis; Adjoint Method; Model Order Reduction

\section{Introduction}

A typical quantity in circuit analysis is the product of voltage difference times the current through an electronic component (power) and, when integrated of time, this reflects the total power that is dissipated. Another time domain problem is the determination of the time moment when a certain unknown, or an expression, crosses a particular value. Such a moment can be the moment at which synchronization is required in co-simulation between a circuit simulator and another simulation tool.

In transient analysis, the adjoint method can be formulated as a convolution of the circuit equations with a carefully constructed function, that, by its nature, requires a backward integration in time of a related DAE (and for which a proper initial value has to be determined). The method has been popularized in $[8,12]$ for linear problems. For more general DAEs the method has been studied in [7] in a more mathematical way. 
In [16] the application to the nonlinear DAEs of circuit equations was studied more closely. Nice applications can be derived for the problem of finding optimal sources in detecting faults in analog circuits [5]. However, in studying sizing problems (in which for instance the physical area of a capacitor has to be taken into account), it appears that especially parameters of capacitors give rise to terms that require additional investigation. Here the effect of the index of the related DAE shows up.

Apart from purposes of optimization, adjoint systems are of interest in determining optimal reduced order models $[3,10]$, in which case a large number of parameters occurs. Because the adjoint systems are linear the equations themselves can be made subject to a reduced order modeling process.

We will describe ways how to calculate sensitivities in a stable and an efficient way.

\section{Transient sensitivity analysis}

Equation (1) is a general Differential-Algebraic Equation (DAE) that can be used to describe how any circuit behaves over a period of time. In Modified Nodal Analysis [12], $\mathbf{x}(t) \in \mathbb{R}^{N}$ is the state vector and represents the node voltages and the currents through voltage sources and inductors, $\mathbf{j}$ and $\mathbf{q}$ are vector functions that describe the current and charge (capacitors) or flux (inductors) behavior. All source values are comprised in $\mathbf{s}(t)$

$$
\frac{\mathrm{d}}{\mathrm{d} t}[\mathbf{q}(\mathbf{x}(t))]+\mathbf{j}(\mathbf{x}(t))=\mathbf{s}(t)
$$

The initial solution at $t=0$, the DC-solution $\mathbf{x}_{\mathrm{DC}}$, satisfies

$$
\mathbf{j}\left(\mathbf{x}_{\mathrm{DC}}\right)=\mathbf{s}(0)
$$

Applying Euler-Backward time integration between time points $t_{n}$ and $t_{n+1}=$ $t_{n}+\Delta t$ enables to calculate $\mathbf{x}^{n+1}$ as approximation at $t_{n+1}$ :

$$
\frac{1}{\Delta t}\left[\mathbf{q}\left(\mathbf{x}^{n+1}\right)-\mathbf{q}\left(\mathbf{x}^{n}\right)\right]+\mathbf{j}\left(\mathbf{x}^{n+1}\right)-\mathbf{s}\left(t_{n+1}\right)=0
$$

A Newton-Raphson procedure involves the coefficient matrix $\mathbf{Y}=\frac{1}{\Delta t} \mathbf{C}+\mathbf{G}$, in which $\mathbf{C}=\partial \mathbf{q} / \partial \mathbf{x}$ and $\mathbf{G}=\partial \mathbf{j} / \partial \mathbf{x}$. Making explicit that the equations and its solution depend on a parameter $\mathbf{p} \in \mathbb{R}^{P}$ we will write

$$
\frac{\mathrm{d}}{\mathrm{d} t}[\mathbf{q}(\mathbf{x}(t, \mathbf{p}), \mathbf{p})]+\mathbf{j}(\mathbf{x}(t, \mathbf{p}), \mathbf{p})=\mathbf{s}(t, \mathbf{p}) .
$$

By adjusting these parameters it is possible to optimize the behavior of a required functionality. The sensitivity of $\mathbf{x}(t, \mathbf{p})$ with respect to $\mathbf{p}$ is denoted by 
$\hat{\mathbf{x}}(t, \mathbf{p}) \equiv \partial \mathbf{x}(t, \mathbf{p}) / \partial \mathbf{p}=\left(\partial x_{i}(t, \mathbf{p}) / \partial p_{j}\right) \in \mathbb{R}^{N \times P}$, and similarly for $\hat{\mathbf{x}}_{\mathrm{DC}}(\mathbf{p})$. After solving (3), and saving of the LU-decomposition of the matrix $\mathbf{Y}=\mathbf{L} \mathbf{U}$, the sensitivity $\hat{\mathbf{x}}^{n+1}(\mathbf{p}) \approx \hat{\mathbf{x}}\left(t_{n+1}, \mathbf{p}\right)$ may be calculated by recursion $[9,11]$

$$
\begin{aligned}
\hat{\mathbf{x}}^{n+1}(\mathbf{p}) & =\mathbf{Y}^{-1} \mathbf{f}, \quad \text { in which } \\
\mathbf{f} & =-\frac{1}{\Delta t}\left[\frac{\partial \mathbf{q}}{\partial \mathbf{p}}^{n+1}-\frac{\partial \mathbf{q}}{\partial \mathbf{p}}\right]-\frac{\partial \mathbf{j}}{\partial \mathbf{p}}^{n+1}+\frac{\partial \mathbf{s}}{\partial \mathbf{p}}^{n+1}+\frac{1}{\Delta t} \mathbf{C} \hat{\mathbf{x}}^{n}(\mathbf{p}) .
\end{aligned}
$$

The vector $\mathbf{f}$ requires $\mathcal{O}\left(P N^{2}\right)$ operations for the last term in addition to $\mathcal{O}(P N)$ evaluations for a term like $\frac{\partial \mathbf{q}}{\partial \mathbf{p}}$ etc... For simplicity we assume full matrices. Solving the system requires an additional $\mathcal{O}\left(P N^{2}\right)$ operations.

A more general basic observation function is denoted by $\mathbf{F}(\mathbf{x}(t, \mathbf{p}), \mathbf{p}) \in \mathbb{R}^{F}$ from which other observation functions can be obtained, like

$$
\mathbf{G}(\mathbf{x}(\mathbf{p}), \mathbf{p})=\int_{0}^{T} \mathbf{F}(\mathbf{x}(t, \mathbf{p}), \mathbf{p}) \mathrm{d} t
$$

From (7) we derive

$$
\frac{\mathrm{d}}{\mathrm{d} \mathbf{p}} \mathbf{G}(\mathbf{x}(\mathbf{p}), \mathbf{p})=\int_{0}^{T}\left(\frac{\partial \mathbf{F}}{\partial \mathbf{x}} \cdot \hat{\mathbf{x}}+\frac{\partial \mathbf{F}}{\partial \mathbf{p}}\right) \mathrm{d} t .
$$

If $\frac{\partial \mathbf{F}}{\partial \mathbf{x}}$ can be determined rather cheaply in (8), the main emphasis in sensitivity analysis is in the efficient calculation of $\hat{\mathbf{x}}$, or even in efficiently calculating the inner-product $\frac{\partial \mathbf{F}}{\partial \mathbf{x}} \cdot \hat{\mathbf{x}}$. Note that, from (5), we derive $\frac{\partial \mathbf{F}}{\partial \mathbf{x}} \cdot \hat{\mathbf{x}}=\frac{\partial \mathbf{F}}{\partial \mathbf{x}} \cdot \mathbf{Y}^{-1} \mathbf{f}=$ $\left[\mathbf{Y}^{-T}\left[\frac{\partial \mathbf{F}}{\partial \mathbf{x}}\right]^{T}\right]^{T} \mathbf{f}$, which can be calculated in $\mathcal{O}\left(\min (F, P) N^{2}+F P N\right)$ operations (in addition to those already mentioned above: the overall leading $N^{2}$-term still has coefficient $P$ ). This is a direct, forward, analysis.

When, additionally, some library for evaluating $\mathbf{q}$, $\mathbf{j}$, or $\mathbf{s}$, does not allow symbolic differentiation, here also a symmetric finite difference will be made (at the cost of two additional evaluations for each quantity $\frac{\mathrm{dq}}{\mathrm{dp}} \approx$ $\left.\frac{\mathbf{q}(\mathbf{p}+\boldsymbol{\Delta} \mathbf{p})-\mathbf{q}(\mathbf{p}-\mathbf{\Delta} \mathbf{p})}{\mathbf{2} \mathbf{p}}\right)$. This means that at each interior time point of (8) the integrand will have an error $\mathcal{O}\left(\|\Delta \mathbf{p}\|^{2}\right.$ ) (assuming this discretization error is dominant). A quadrature rule like the Trapezoidal Rule adds up to $\mathcal{O}\left(\|\Delta \mathbf{p}\|^{2} / \Delta t\right)$ leading to $\|\Delta \mathbf{p}\|=o(\sqrt{\Delta t})$ if $\Delta t \rightarrow 0$ and no persistent errors in sensitivities are wanted.

In the sequel, we now consider an approach based on (backward) adjoint integration [7]. We differentiate (4) w.r.t. $\mathbf{p}$ and multiply the result with a function $\lambda^{\star}(t) \in \mathbb{R}^{F \times N}$ (in which the ${ }^{\star}$ means transpose), yielding

$$
\begin{aligned}
0 & =\int_{0}^{T} \lambda^{\star}(t)\left[\frac{\mathrm{d}}{\mathrm{d} t} \frac{\mathrm{d} \mathbf{q}}{\mathrm{d} \mathbf{p}}+\frac{\mathrm{d} \mathbf{j}}{\mathrm{d} \mathbf{p}}-\frac{\partial \mathbf{s}}{\partial \mathbf{p}}\right] \mathrm{d} t \\
& =\left.\left[\lambda^{\star}(t) \frac{\mathrm{d} \mathbf{q}}{\mathrm{d} \mathbf{p}}\right]\right|_{0} ^{T}+\int_{0}^{T}\left[-\frac{\mathrm{d} \lambda^{\star}}{\mathrm{d} t} \frac{\mathrm{d} \mathbf{q}}{\mathrm{d} \mathbf{p}}+\lambda^{\star}\left(\frac{\mathrm{d} \mathbf{j}}{\mathrm{d} \mathbf{p}}-\frac{\partial \mathbf{s}}{\partial \mathbf{p}}\right)\right] \mathrm{d} t \\
& =\left.\left[\lambda^{\star}(t) \frac{\mathrm{d} \mathbf{q}}{\mathrm{d} \mathbf{p}}\right]\right|_{0} ^{T}+\int_{0}^{T}\left[-\frac{\mathrm{d} \lambda^{\star}}{\mathrm{d} t}\left(\frac{\partial \mathbf{q}}{\partial \mathbf{x}} \cdot \frac{\partial \mathbf{x}}{\partial \mathbf{p}}+\frac{\partial \mathbf{q}}{\partial \mathbf{p}}\right)+\lambda^{\star}\left(\frac{\partial \mathbf{j}}{\partial \mathbf{x}} \cdot \frac{\partial \mathbf{x}}{\partial \mathbf{p}}+\frac{\partial \mathbf{j}}{\partial \mathbf{p}}-\frac{\partial \mathbf{s}}{\partial \mathbf{p}}\right)\right] \mathrm{d} t
\end{aligned}
$$




$$
\begin{aligned}
& =\left.\left[\lambda^{\star}(t) \frac{\mathrm{d} \mathbf{q}}{\mathrm{d} \mathbf{p}}\right]\right|_{0} ^{T}+\int_{0}^{T}\left[-\frac{\mathrm{d} \lambda^{\star}}{\mathrm{d} t}\left(\mathbf{C} \hat{\mathbf{x}}+\frac{\partial \mathbf{q}}{\partial \mathbf{p}}\right)+\lambda^{\star}\left(\mathbf{G} \hat{\mathbf{x}}+\frac{\partial \mathbf{j}}{\partial \mathbf{p}}-\frac{\partial \mathbf{s}}{\partial \mathbf{p}}\right)\right] \mathrm{d} t \\
& =\left.\left[\lambda^{\star}(t) \frac{\mathrm{d} \mathbf{q}}{\mathrm{d} \mathbf{p}}\right]\right|_{0} ^{T}+\int_{0}^{T}\left[-\left(\frac{\mathrm{d} \lambda^{\star}}{\mathrm{d} t} \mathbf{C}-\lambda^{\star} \mathbf{G}\right) \hat{\mathbf{x}}-\frac{\mathrm{d} \lambda^{\star}}{\mathrm{d} t} \frac{\partial \mathbf{q}}{\partial \mathbf{p}}+\lambda^{\star}\left(\frac{\partial \mathbf{j}}{\partial \mathbf{p}}-\frac{\partial \mathbf{s}}{\partial \mathbf{p}}\right)\right] \mathrm{d} t(10)
\end{aligned}
$$

This result holds for any $\lambda^{\star}$. We now consider some choices.

\subsection{Backward, adjoint sensitivity for $\frac{d}{d p} G(x(p), p)$}

In (8) we encounter the product $\frac{\partial \mathbf{F}}{\partial \mathbf{x}} \cdot \hat{\mathbf{x}}$. Equation (10) will enable us to get rid of the $\hat{\mathbf{x}}$, which does not need to be calculated explicitly. We choose $\lambda(t) \in$ $\mathbb{R}^{N \times F}$ appropriately and require that $\lambda(t)$ satisfies the linear 'adjoint' DAE (we assume the index 1 case, which is not trivial [4]).

$$
\mathbf{C}^{\star} \frac{\mathrm{d} \lambda}{\mathrm{d} t}-\mathbf{G}^{\star} \lambda=-\left(\frac{\partial \mathbf{F}}{\partial \mathbf{x}}\right)^{\star}
$$

This does not yet make $\lambda(t)$ unique, because we did not specify the initial value yet. But we are now able to express $\frac{\partial \mathbf{F}}{\partial \mathbf{x}} \cdot \hat{\mathbf{x}}$ in terms of $\left[\mathbf{C}^{\star} \frac{\mathrm{d} \lambda}{\mathrm{d} t}-\mathbf{G}^{\star} \lambda\right] \cdot \hat{\mathbf{x}}$, after which we can apply (10).

$$
\begin{aligned}
\frac{\mathrm{d}}{\mathrm{d} \mathbf{p}} \mathbf{G}(\mathbf{x}(\mathbf{p}), \mathbf{p}) & =\int_{0}^{T}\left(\frac{\partial \mathbf{F}}{\partial \mathbf{x}} \cdot \hat{\mathbf{x}}+\frac{\partial \mathbf{F}}{\partial \mathbf{p}}\right) \mathrm{d} t \\
& =\int_{0}^{T}\left(\left[-\frac{\mathrm{d} \lambda^{\star}}{\mathrm{d} t} \mathbf{C}+\lambda^{\star} \mathbf{G}\right] \cdot \hat{\mathbf{x}}+\frac{\partial \mathbf{F}}{\partial \mathbf{p}}\right) \mathrm{d} t \\
& =-\left.\left[\lambda^{\star}(t) \frac{\mathrm{d} \mathbf{q}}{\mathrm{d} \mathbf{p}}\right]\right|_{t=0} ^{T}+\int_{0}^{T}\left(\frac{\mathrm{d} \lambda^{\star}}{\mathrm{d} t} \frac{\partial \mathbf{q}}{\partial \mathbf{p}}-\lambda^{\star}\left(\frac{\partial \mathbf{j}}{\partial \mathbf{p}}-\frac{\partial \mathbf{s}}{\partial \mathbf{p}}\right)+\frac{\partial \mathbf{F}}{\partial \mathbf{p}}\right) \mathrm{d} t .
\end{aligned}
$$

The first term involves $\hat{\mathbf{x}}(0)=\hat{\mathbf{x}}_{\text {DC }}$, which we know. However, if $\lambda^{\star}(T) \frac{\partial \mathbf{q}}{\partial \mathbf{x}}(T) \neq$ 0 , one still needs $\hat{\mathbf{x}}(T)$, which we did not want to determine explicitly. Hence good choices to define the initial conditions for $\lambda(t)$ are

$$
\begin{aligned}
\mathbf{C}(T) & =\frac{\partial \mathbf{q}}{\partial \mathbf{x}}(T) \neq 0 \Longrightarrow \lambda(T)=0, \text { or if } \\
\mathbf{C}(t) & =\frac{\partial \mathbf{q}}{\partial \mathbf{x}}(t) \equiv 0 \Longrightarrow \lambda(T) \equiv \lambda_{\mathrm{DC}}, \text { with }-\mathbf{G}^{\star} \lambda_{\mathrm{DC}}=-\left.\left(\frac{\partial \mathbf{F}}{\partial \mathbf{x}}\right)^{\star}\right|_{t=0}
\end{aligned}
$$

Note that (13) is only allowed for DAEs of index up to 1 . With this choice (12) simplifies to

$$
\begin{aligned}
\frac{\mathrm{d}}{\mathrm{d} \mathbf{p}} \mathbf{G}(\mathbf{x}(\mathbf{p}), \mathbf{p})= & \lambda^{\star}(0)\left[\frac{\partial \mathbf{q}}{\partial \mathbf{x}}(0) \cdot \hat{\mathbf{x}}_{\mathrm{DC}}+\frac{\partial \mathbf{q}}{\partial \mathbf{p}}(0)\right]+ \\
& \int_{0}^{T}\left(\frac{\mathrm{d} \lambda^{\star}}{\mathrm{d} t} \frac{\partial \mathbf{q}}{\partial \mathbf{p}}-\lambda^{\star}\left(\frac{\partial \mathbf{j}}{\partial \mathbf{p}}-\frac{\partial \mathbf{s}}{\partial \mathbf{p}}\right)+\frac{\partial \mathbf{F}}{\partial \mathbf{p}}\right) \mathrm{d} t .
\end{aligned}
$$


The circuit that describes the adjoint system (11) is described in more details in $[8,12]$. There the above expression is derived by conservation properties based on the Kirchhoff Laws which imply Tellegen's Theorem. Note that (9) is just the time integral of inner-products of "branch-currents AdjointCircuit ${ }^{*}$ branch-voltages OriginalCircuit".

The formulation used above follows [7], but reveals more closely the effect of a non-trivial function $\mathbf{q}$ in (12) that explicitly depends on $\mathbf{p}$. By this, also the velocity $\frac{\mathrm{d} \lambda^{\star}}{\mathrm{d} t}$ will be needed. Already a linear function $\mathbf{q}(\mathbf{x}, \mathbf{p})=\mathbf{C}(\mathbf{p}) \mathbf{x}$ requires this. Because of the DAE-nature of the problem (1), in [16], $\frac{\mathrm{d} \lambda^{\star}}{\mathrm{d} t}$ was estimated by symmetric finite differences in the interior of the interval and with one-sided approximations at the boundaries. When, as before, a library for evaluating $\mathbf{q}, \mathbf{j}$, or $\mathbf{s}$, does not allow symbolic differentiation, here also a symmetric finite difference will be made (at the cost of two additional evaluations for each quantity). This means that at each interior time point the integrand will have an error $\mathcal{O}\left(\Delta t^{2}+\|\Delta \mathbf{p}\|^{2}\right)$. Any quadrature rule (like the Trapezoidal Rule) may add these errors up to an error $\mathcal{O}\left(\Delta t+\|\Delta \mathbf{p}\|^{2} / \Delta t\right)$ which is additional to the error of the quadrature rule, which means that one will require $\|\Delta \mathbf{p}\|=\mathcal{O}(\Delta t)$ (note that a one-sided difference will even need $\left.\|\Delta \mathbf{p}\|=\mathcal{O}\left(\Delta t^{2}\right)\right)$. It also shows that the Trapezoidal Rule may not give better results than simple, first order, Euler integration.

System (11) can be determined by integrating backwards in time after $\mathbf{x}(t, \mathbf{p})$ has been determined in the nominal analysis. This backward time integration of (11) requires Jacobian matrices $\frac{1}{\Delta t} \mathbf{C}+\mathbf{G}$ (assuming Euler backwards), similar as in the forward analysis. When the same step sizes are used and the LU-decompositions of the Jacobian matrices at the converged values have been saved from the nominal analysis, the transposed decompositions can be reused for the Jacobians when integrating (11) (one may also save approximative inverse matrices, or preconditioning matrices). However we will assume that one will re-decompose them during the backward integration.

Note that in [6] it is remarked that if the same time step is used in the forward (for $\mathbf{x}$ ) and backward analysis (for $\lambda$ ) this may give rise to very inaccurate solutions for $\lambda$. This general step size approach introduces effects due to interpolation (effects which we have not yet studied).

Let $\mathcal{W}=\mathcal{O}\left(N^{\alpha}\right)$ represent the number of operations for the LU-decompositions with $1 \leqslant \alpha \leqslant 2$ for sparse systems and $\alpha=3$ for full systems. Note that $\lambda^{\star}(0) \frac{\partial \mathbf{q}}{\partial \mathbf{x}}(0) \cdot \hat{\mathbf{x}}_{\mathrm{DC}}=\left[\mathbf{C}^{\star} \lambda(0)\right]^{*} \hat{\mathbf{x}}_{\mathrm{DC}}$, which can be handled similarly as in adjoint sensitivity analysis in the DC-problem, without explicitly calculating $\hat{\mathbf{x}}_{\mathrm{DC}}$, in $\mathcal{W}+\mathcal{O}\left(\min (F, P) N^{2}+F P N\right)$ operations (assuming that $\mathbf{G}$ has been decomposed again at $t=0$ ). Each time integration step of (11) requires $\mathcal{W}+\mathcal{O}\left(F N^{2}\right)$ operations, after which the integrand in (15) at each time point requires $\mathcal{O}(P N+F P)$ evaluations and $\mathcal{O}(F P N)$ additional operations. In practise $F \ll P$, which (apart from the $\mathcal{W}$ term) makes (15) more efficient as the direct, forward method for (5)-(6).

Reduction of the $\mathcal{W}$ term is discussed in Section 2.2. 


\subsection{MOR applied to the global adjoint sensitivity equations}

The main burden of the backward adjoint sensitivity equations still is the $\mathcal{W}=\mathcal{O}\left(N^{\alpha}\right)$ work needed for the LU-decompositions in the case $\alpha=3$ when integrating backwards in time for the adjoint equations for $\lambda(t)$. In order to reduce this we observe that in the interior we only need to know $\lambda(t)$ for coordinates where $\frac{\partial \mathbf{q}}{\partial \mathbf{p}}, \frac{\partial \mathbf{j}}{\partial \mathbf{p}}$, and $\frac{\partial \mathbf{s}}{\partial \mathbf{p}}$ are non trivial. However, at $t=0$ also for the nontrivial rows of $\frac{\partial \mathbf{q}}{\partial \mathbf{x}}(0)$ coordinates of $\lambda(t)$ should be known (which may significantly increase the number of needed coordinates). More precisely, in (15) a term like $\lambda^{\star}(0) \frac{\partial \mathbf{q}}{\partial \mathbf{x}}(0) \cdot \hat{\mathbf{x}}_{\mathrm{DC}} \in \mathbb{R}^{F \times P}$ shows that the total number of output is $r^{\prime}=F \times P$, and when $r^{\prime} \ll N$, one may think to apply MOR.

We observe that there is a tentative opportunity to apply Proper Orthogonal Decomposition (POD) $[1,13]$, since the forward time integration to determine $\mathbf{x}(t)$ delivered a nice series of snapshots $\left\{\mathbf{x}\left(t_{0}\right), \ldots, \mathbf{x}\left(t_{N}\right)\right\}$ (and, even cheaply, also of $\left.\frac{\partial \mathbf{F}}{\partial \mathbf{x}}\left(t_{k}\right)\right)$. With POD a matrix $\mathbf{V} \in \mathbb{R}^{N \times r}$ is found such that $\mathbf{x} \approx \mathbf{V} \tilde{\mathbf{x}}$, $\tilde{\mathbf{x}} \in \mathbb{R}^{r}, r \ll N$, in which $\mathbf{V}$ is time independent and similar snapshots could have been obtained from a modified problem

$$
\frac{\mathrm{d}}{\mathrm{d} t}\left[\mathbf{V}^{T} \mathbf{q}(\mathbf{V} \tilde{\mathbf{x}}(t))\right]+\mathbf{V}^{T} \mathbf{j}(\mathbf{V} \tilde{\mathbf{x}}(t))=\mathbf{V}^{T} \mathbf{s}(t) .
$$

For (16) the matrices are of size $r \times r$, which indicates a nice option for MOR. In this paper we apply the $\mathbf{V}^{\mathbf{T}}, \mathbf{V}$ matrices from the POD directly to the adjoint problem (11). Note that in this approach we can neglect the dependency of $\mathbf{V}$ on $\mathbf{p}$. For each $\mathbf{p}$ one integrates (1) and calculates the snapshots, resulting in a POD Model Order reduction projection matrix $\mathbf{V}$. With $\mathbf{V}$ we can reduce the system of DAEs for $\lambda$ (in which only the error due to POD matters) resulting in $\lambda_{P O D}(t) \sim \lambda(t)$ (please see [13] for error estimates). Note that POD can also be used to reduce (1) itself. In this case the dependency of $\mathbf{V}$ on $\mathbf{p}$ introduces an additional error in the procedure that can not be neglected. This last approach could further reduce costs and has to be studied further. In [2] it was shown that (in general) POD not directly applies to DAEs. Here a Least-Square POD remedy was introduced that can be applied to the linear DAE (11).

Alternatively to POD, during the forward integration one could additionally determine projection matrices for the Trajectory Piecewise-Linear Method $[14,15]$. Next, similarly as to the POD case, a global matrix $\mathbf{V}$ is determined that allows for Piecewise-Linear MOR. In this case the reduced DAE problems can directly be solved [15]. 


\section{Results}

\begin{tabular}{|c|c|c|}
\hline $\mathbf{p}=l_{R}(m)$ & $\mathbf{d G} / \mathbf{d p}$ & $\mathbf{d G} / \mathbf{d p}$ withPOD \\
\hline \hline 0.02 & $-0.235 \times 10^{-10}$ & $-0.235 \times 10^{-10}$ \\
\hline 0.021 & $-0.247 \times 10^{-10}$ & $-0.247 \times 10^{-10}$ \\
\hline 0.022 & $-0.256 \times 10^{-10}$ & $-0.256 \times 10^{-10}$ \\
\hline
\end{tabular}

Table 1. Sensitivities, where $\mathbf{p}$ is the parameter, in this case the parameter is the length of the second resistor. Units are $\left(W t m^{-1}\right)$

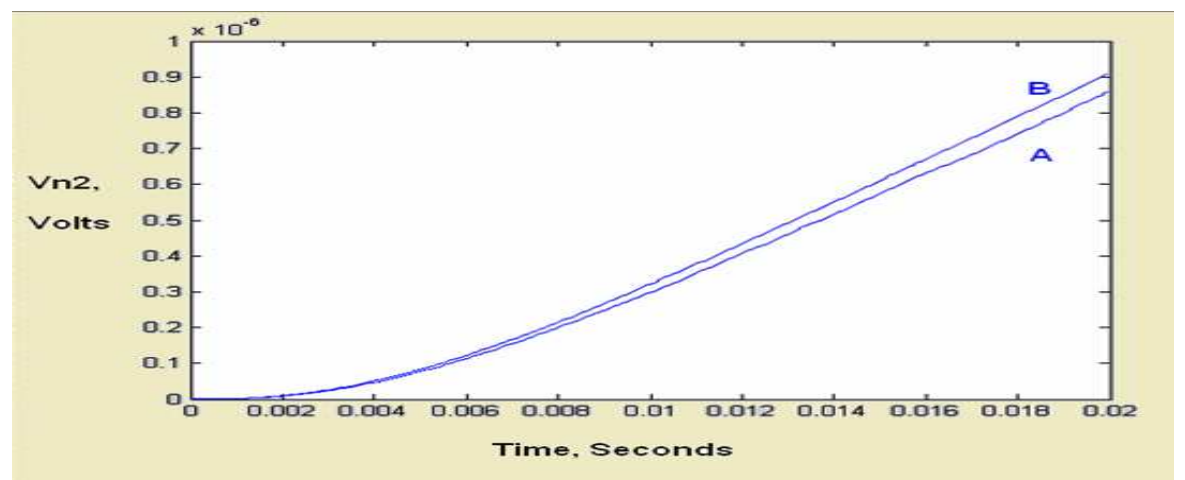

Fig. 1. Voltage differences for successive sensitivity values, node 2

Equation (12) was implemented in Matlab for a simple circuit and the sensitivities of the energy $\mathbf{G}$ dissipated for a resistor $\mathbf{R}=\mathbf{R}\left(l_{R}\right)$ were observed while changing the length parameter $l_{R}$

$$
\mathbf{G}=\int_{0}^{T} \mathbf{I}(\mathbf{R}) * \mathbf{V}(\mathbf{R}) d t
$$

To see the dependency of $\mathbf{V}_{\mathbf{R}}$ for different values of $\mathbf{p}$, in Fig.1. we plotted,

A: $\left(\mathbf{V}_{\mathbf{R}}(\mathbf{p}=0.021)-\mathbf{V}_{\mathbf{R}}(\mathbf{p}=0.020)\right)(t)$

B: $\left(\mathbf{V}_{\mathbf{R}}(\mathbf{p}=0.022)-\mathbf{V}_{\mathbf{R}}(\mathbf{p}=0.021)\right)(t)$.

In Table 1 the sensitivity $\mathbf{d G} / \mathbf{d p}$, calculated by the backward adjoint method, are shown for 3 different values of $\mathbf{p}$. To test POD we created a larger, equivalent system by splitting one resistor in to a number of smaller resistors. Doing this enabled us to compare the sensitivity of our observed resistor in the reduced large system with the sensitivity in the original system. We generated 
our projection matrices by applying the singular value decomposition on the new state snapshot matrix for the enlarged problem. Indeed the number of nontrivial singular values for both systems was the same. We applied the projection matrices to reduce only the backward adjoint calculation steps. The size of the matrices was considerably reduced and the calculated sensitivities from the reduced system were exact to at least 3 significant figures, please see Table 1 for sensitivity results for the POD and for the non POD approach.

\section{Conclusions}

The backward, adjoint sensitivity methods are immediately attractive when the original DAE (1) is linear and when the number of parameters $P \gg 1$. Direct forward and backward adjoint approaches impose different accuracy conditions to finite difference approximations. The direct forward method exploits the re-use of LU-decompositions. The backward adjoint methods becomes more of interest when MOR can be applied, or when otherwise approximate LU-decompositions could have been saved during the forward time integration. In these cases they can outperform the direct forward method when the number of parameters $P$ is large (but still smaller than $N$, usually $F \ll P)$.

We have shown that applying POD MOR to the backward adjoint step is possible and works very well. MOR techniques can also be used to reduce the effort in sensitivity calculation in the forward analysis. In future we want to study more closely the application of our sensitivity calculations to larger circuits and with more industrial relevance. We also want to consider the effect of different time stepping in forward and backward analysis. And, finally, we want to study the sensitivity of the reduced DAE (16) in which $\mathbf{V}=\mathbf{V}(\mathbf{p})$ depends on $\mathbf{p}$ as well.

\section{References}

1. P. Astrid: Reduction of process simulation models: a proper orthogonal decomposition approach, PhD-Thesis, Department of Electrical Engineering, Eindhoven University of Technology, 2004

2. P. Astrid, A. Verhoeven: Application of least squares MPE technique in the reduced order modeling of electrical circuits, TU Eindhoven, Center for Analysis, Scientific Computing and Applications, CASA Report 11, 2006

3. O. Balima, Y. Favennec, M. Girault, D. Petit: Comparison between the modal identification method and the POD-Galerkin method for model reduction in nonlinear diffusion systems, Int. J.Numer. Meth. Engng., Vol. 67, pp. 895-915, 2006.

4. K. Balla, R. März : Linear differential-algebraic equations of index-1 and their adjoint equations, Results in Maths, Vol.37, pp.12-35, 2000.

5. B. Burdiek: Generation of optimal test stimuli for nonlinear analog circuits using nonlinear programming and time-domain sensitivities, Proc. DATE 2001, Munchen, pp. 603-608.

6. B. Burdiek: Zur Berechnung von Testsignalen für nichtlineare analoge Schaltungen unter Verwendung von Methoden der Optimalsteurungstheorie, Shaker, Achen, 2005 (PhD Thesis Univ. of Haannover, 2005).

7. Y. Cao, S. Li, L. Petzold, R. Serban: Adjoint sensitivity for differential-algebraic equations: the adjoint DAE system and its numerical solution, SIAM J. Sci. Comput., Vol. 24-3, pp. 1076-1089, 2002. 
8. A.R. Conn, P.K. Coulman, R.A. Haring, G.L. Morrill, C. Visweswariah, C.W. Wu: JiffyTune: circuit optimization using time-domain sensitivities, IEEE Trans. on CAD of ICs and Systems, Vol. 17-12, pp. 1292-1309, 1998

9. L. Daldoss, P. Gubian, M. Quarantelli: Multiparameter time-domain sensitivity computation, IEEE Trans. on Circuits and Systems - I: Fund. Theory and Applics, Vol. 48-11, pp. 1296-1307, 2001.

10. Y. Favennec, M. Girault, D. Petit: The adjoint method coupled with the modal identification method for nonlinear model reduction, Inverse Probl. in Science and Engng., Vol. 14, No. 3 , 153-170, 2006.

11. D.E. Hocevar, P. Yang, T.N. Trick, B.D. Epler: Transient sensitivity computation for MOS FET circuits, IEEE Trans. on CAD of Integr. Circuits and Systems, Vol. CAD-4, Nr. 4, pp. 609-620, 1985.

12. L.T. Pillage, R.A. Rohrer, C. Visweswariah: Electronic circuit and system simulation methods, McGraw-Hill, Inc, New York, USA, ISBN 0070501696, 1994.

13. M. Rathinam, L.R. Petzold: A new look at proper orthogonal decomposition, SIAM J. Numer. Analysis, Vol. 41-5, pp. 1893-1925, 2003.

14. M.J. Rewienski: A trajectory piecewise-linear approach to model order reduction of nonlinear dynamical systems, PhD-Thesis, Massachutes Institute of Technology, 2003.

15. T. Voss: Model reduction for nonlinear differential algebraic equations, MSc. Thesis University of Wuppertal, 2005; Unclassified Report PR-TN-2005/00919, Philips Research Laboratories, 2005.

16. H. Xu: Transient Sensitivity Analysis in Circuit Simulation, MSc-Thesis, Department of Mathematics and Computing Science, Eindhoven University of Technology, 2004. [Online via author search at Library at http://w3.win.tue.nl/en/] 